\title{
Fluorodeoxyglucose uptake in laryngeal carcinoma is associated with the expression of glucose transporter-1 and hypoxia-inducible-factor-1 $\alpha$ and the phosphoinositide 3-kinase/protein kinase B pathway
}

\author{
KUI ZHAO ${ }^{1}$, SHU-YE YANG ${ }^{1}$, SHUI-HONG ZHOU ${ }^{2}$, MENG JIE DONG ${ }^{1}$, \\ YANG-YANG BAO ${ }^{2}$ and HONG-TIAN YAO ${ }^{3}$ \\ Departments of ${ }^{1}$ PET Center, ${ }^{2}$ Otolaryngology and ${ }^{3}$ Pathology, The First Affiliated Hospital, \\ College of Medicine, Zhejiang University, Hangzhou, Zhejiang 310003, P.R. China
}

Received July 14, 2013; Accepted January 23, 2014

DOI: $10.3892 / 01.2014 .1877$

\begin{abstract}
High fluorodeoxyglucose (FDG) uptake by human carcinomas, including head and neck cancers, is associated with a poor prognosis. Glucose transporter-1 (Glut-1) is believed to be an intrinsic marker of hypoxia in malignant tumors. The expression of hypoxia-inducible factor- $1 \alpha$ (HIF-1 $\alpha$ ) and correlated target genes, including Glut-1, is regulated by the phosphoinositide 3-kinase/protein kinase $\mathrm{B}$ (PI3K/Akt) pathway. However, it remains unclear whether the PI3K/Akt signaling pathway is involved in regulating FDG uptake directly. In the present study, 24 consecutive patients with laryngeal carcinoma were examined pre-operatively and the standardized uptake values (SUV) of the laryngeal carcinomas were determined. Glut-1, HIF-1 $\alpha$, PI3K and phosphorylated-Akt (p-Akt) expression was detected by immunohistochemical staining of paraffin sections from the tumor specimens. Associations among $\mathrm{SUV}_{\max }$, Glut-1, HIF-1 $\alpha$, PI3K and p-Akt protein expression and the other clinical parameters were analyzed. The univariate analyses revealed a significantly shorter survival time along with higher HIF-1 $\alpha(\mathrm{P}=0.018)$ and PI3K $(\mathrm{P}=0.008)$ expression, but the survival time was not significantly correlated with Glut-1 or p-Akt expression. The multivariate analysis demonstrated that higher $\operatorname{SUV}_{\max }(\mathrm{P}=0.043)$ and PI3K expression $(\mathrm{P}=0.012)$ were significantly correlated with a poor survival time. Spearman's rank
\end{abstract}

Correspondence to: Dr Shui-Hong Zhou, Department of Otolaryngology, The First Affiliated Hospital, College of Medicine, Zhejiang University, 79 Qingchun Road, Hangzhou, Zhejiang 310003, P.R. China

E-mail: zhouyunzhoush@163.com

Key words: laryngeal carcinoma, glucose transporter-1, hypoxia inducible factor-1 $\alpha$, phosphoinositide 3-kinase/protein kinase B signal pathway, fluorodeoxyglucose, prognosis analysis showed significant correlations between $\mathrm{SUV}_{\max }$ and HIF-1 $\alpha(\mathrm{r}=0.577 ; \mathrm{P}=0.003), \mathrm{PI} 3 \mathrm{~K}(\mathrm{r}=1.0 ; \mathrm{P}<0.0001)$ and $\mathrm{p}-\mathrm{Akt}(\mathrm{r}=0.577 ; \mathrm{P}=0.003)$ expression. $\mathrm{PI} 3 \mathrm{~K}$ was associated with poorly- and moderately-differentiated laryngeal carcinoma $(\mathrm{P}=0.012)$. In conclusion, a high $\mathrm{SUV}_{\max }$ indicates a poor prognosis for laryngeal carcinoma. Also, a high $\mathrm{SUV}_{\max }$ may be associated with the increased expression of Glut-1, HIF-1 $\alpha$, PI3K and p-Akt.

\section{Introduction}

$\left[{ }^{18} \mathrm{~F}\right]$-2-fluoro-2-deoxy-D-glucose $\left({ }^{18} \mathrm{~F}\right.$-FDG) positron emission tomography/computed tomography (PET/CT) imaging has been used widely for the diagnosis, pre-operative staging, restaging, prognostic prediction and detection of unknown primary tumors (1-3). Increased uptake of FDG, a glucose analog, directly reflects the higher glucose metabolic rate in malignant tumor cells compared with their non-malignant counterparts $(4,5)$.

Numerous mechanisms have been proposed for the accelerated glucose use in growing tumors and in transformed and malignant cells, including passive diffusion, $\mathrm{Na}^{+}$-dependent glucose transport, oncogenes and facilitative glucose transporter (Glut) (6-8). Numerous studies (9-12), including our previous study (6), have revealed that Glut-1 plays a significant role in malignant glucose metabolism and that it may contribute to increased FDG uptake. Certain studies have considered Glut-1 as a possible intrinsic marker of hypoxia in malignant tumors $(13,14)$. Hypoxia of solid tumors has been associated with therapy resistance and a poor clinical prognosis. Biological markers that predict tumor hypoxia may be useful for selecting treatments and predicting patient prognosis (9). Certain studies have demonstrated that FDG indirectly reflects the hypoxic status of malignant tumors $(9,10,15-18)$ since FDG is associated with hypoxia markers, including Glut-1, phosphoinositide 3-kinase (PI3K) and hypoxia inducible factor-1 $\alpha$ (HIF-1 $\alpha)(2,9,10,15-17)$. High FDG uptake by human carcinomas is also associated with a poor prognosis $(9,19)$. Our previous study identified that the 
FDG uptake detected by single photon emission CT in head and neck cancers was associated with increased Glut-1 expression (20). Our recent preliminary study also revealed that the maximum standardized uptake value $\left(\mathrm{SUV}_{\max }\right)$ of PET/CT in the cervical lymph nodes predicted cervical metastasis of a carcinoma from an unknown primary tumor (3). There is certain controversy, however, as certain studies did not find any association between FDG uptake and the hypoxic status of specific carcinomas $(21,22)$. The expression of HIF- $1 \alpha$ and the correlated target genes, including Glut-1, is regulated by the $\mathrm{PI} 3 \mathrm{~K} /$ protein kinase B (Akt) pathway $(23,24)$. However, it remains unclear whether the PI3K/Akt signal pathway is involved in the regulation of FDG uptake $(15,16,25,26)$. Consequently, the association between FDG and hypoxia markers must be studied further. Therefore, the correlation between FDG uptake and the hypoxia markers, Glut-1, HIF-1 $\alpha$, PI3K and Akt, was investigated in the present study using immunohistochemistry to clarify whether FDG-PET can be used to predict tumor hypoxia and the prognosis of patients with laryngeal carcinoma.

\section{Materials and methods}

Patients. Using PET/CT, 24 consecutive patients [21 male and 3 female; mean age, 60.8 years (range, $37-80$ years)] with laryngeal carcinoma were examined pre-operatively. No patient received tumor-directed therapy pre-operatively. The institutional review board of The First Affiliated Hospital, College of Medicine, Zhejiang University (Hangzhou, Zhejiang, China) approved the present study, and written informed consent was obtained from each patient prior to inclusion.

$P E T / C T$. Whole-body imaging was performed using a combined PET/CT scanner (Biograph Sensation 16; Siemens Medical, Erlangen, Germany). Following $\geq 4-6 \mathrm{~h}$ of fasting, the patients were administered an intravenous injection of FDG at a dosage of 5.5-7.4 MBq (0.15-0.20 mCi)/kg. The blood glucose levels of the patients were checked prior to the ${ }^{18}$ F-FDG injection. Data acquisition started 60-90 min after FDG administration following routine procedures: Low-dose CT was performed from head to mid-thigh at $120 \mathrm{kV}, 50 \mathrm{~mA}$ and with a $2-5-\mathrm{mm}$ slice thickness matching the PET section, followed by three-dimensional PET scanning at $2 \mathrm{~min} /$ bed position. The PET emission scan was performed from the subcranial region to mid-thigh, whereas the brain scan required another bed position. The PET images were reconstructed iteratively using ordered subset Syngo Speaking (Wizard Workstation; Siemens Medical Solutions USA, Inc., Malvern, PA, USA) with CT-based attenuation correction. PET, CT and fused PET/CT images were generated and reviewed on a workstation. The $\mathrm{SUV}_{\max }$ was assessed from the predominant lesion and calculated based on the amount of injected ${ }^{18} \mathrm{~F}-\mathrm{FDG}$ and the body weight: SUV $=$ [decay corrected activity $(\mathrm{kBq}) /$ tissue volume $(\mathrm{ml})$ ] / [injected FDG activity $(\mathrm{kBq}) /$ body weight $(\mathrm{g})]$ (3). The PET/CT images were interpreted independently by two experienced PET/CT physicians blinded to the clinical data. Any variations between the interpretation of the data were settled by consensus.
Immunohistochemical analysis and evaluation. For the immunohistochemical evaluation, paraffin blocks of formalin-fixed specimens were obtained via biopsies of the predominant lesions in each subject. Formalin-fixed, paraffin-embedded tissue blocks from the primary lesions were cut into $4-\mu \mathrm{m}$ sections, and representative sections were analyzed immunohistochemically (EliVision ${ }^{\mathrm{TM}}$ Plus IHC kit; Fuzhou Maixin Biotechnology Development, Co., Ltd., Fuzhou, Fuijan, China), using a rabbit polyclonal antibody against Glut-1 (1:50), a mouse monoclonal antibody against HIF-1 $\alpha$ (1:100), a rabbit monoclonal antibody against PI3K (1:100) and a rabbit polyclonal antibody against p-AKT (1:50) (all Santa Cruz Biotechnology, Inc., Santa Cruz, CA, USA) (25). Briefly, antigen retrieval was performed subsequent to the sections being deparaffinized with xylene and dehydrated through an ethanol series. Endogenous peroxidase activity was blocked by incubating the slides in $1.5 \%$ hydrogen peroxide in absolute methanol at room temperature for $10 \mathrm{~min}$. Primary antibodies were applied for $1 \mathrm{~h}$ at room temperature, followed by use of $50 \mu \mathrm{l}$ polymer enhancer for $20 \mathrm{~min}$ and $50 \mu \mathrm{l}$ polymerized horseradish peroxidase-anti-mouse immunoglobulin $\mathrm{G}$ for $30 \mathrm{~min}$. The reaction products were visualized using a diaminobenzidine kit (Fuzhou Maixin Biological Technology, Ltd.), and then the sections were counterstained with hematoxylin and eosin, dehydrated and examined under a light microscope. Tris-buffered saline was used instead of the primary antibody for the negative controls. Erythrocytes, which were present in all sections, served as internal controls for Glut-1 to confirm the constant immunostaining intensity.

Glut-1, HIF-1 $\alpha$, PI3K and p-Akt were evaluated by the same investigator who was blinded to the clinical and follow-up data. Glut-1 expression was considered positive only if distinct membrane staining was present. HIF-1 $\alpha, \mathrm{PI} 3 \mathrm{~K}$ and p-AKT proteins were observed in the nucleus and cytoplasm. Protein analysis was performed in 10 random high fields, and 100 tumor cells were counted in each high-power field for each case and for all antibodies used. The percentage of positive cells was calculated by dividing the number of positive tumor cells by the total number of tumor cells counted. A sample was considered negative if $<25 \%$ of the cells were stained (2).

Follow-up. The patients were asked to come back for follow-up at 1-, 3-, and 6-month intervals in the first, second and third to fifth years after the initial surgery, respectively, and then annually thereafter. Follow-up consisted of a routine physical examination, strobolarynoscopy and CT or magnetic resonance imaging of the primary site. Patient follow-up was reported up to the date of the last visit to the clinic.

Statistical analyses. The statistical analyses were performed using SPSS for Windows, version 19.0 (SPSS, Inc., Chicago,

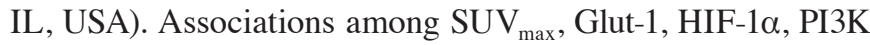
and p-Akt protein expression and the other pre-treatment parameters were analyzed using the $\chi^{2}$ test and Fisher's exact test. Logistic regression was used for the multivariate analysis. $\mathrm{P}<0.05$ was deemed to indicate statistically significant differences, and the correlation analysis was performed using Spearman's correlation. 
Table I. Clinicopathological characteristics of 24 patients with laryngeal carcinoma.

\begin{tabular}{|c|c|c|c|c|c|c|c|c|c|c|c|c|}
\hline $\begin{array}{l}\mathrm{Pt} \\
\text { no. }\end{array}$ & $\begin{array}{c}\text { Gender/age, } \\
\text { years }\end{array}$ & Pathology & $\mathrm{HG}$ & Site & TNM & Treatment & $\begin{array}{l}\text { Follow-up, } \\
\text { months }\end{array}$ & Glut-1 & HIF & PI3K & $\mathrm{p}-\mathrm{Akt}$ & $\mathrm{SUV}_{\max }$ \\
\hline 1 & $\mathrm{M} / 58$ & $\mathrm{NC}$ & PD & Subglottic & $\mathrm{T} 2 \mathrm{~N} 2 \mathrm{M} 0$ & $\begin{array}{c}\text { TL+SND } \\
+ \text { PRT }\end{array}$ & $\begin{array}{l}6 \mathrm{R}, \\
18 \mathrm{DOD}\end{array}$ & + & + & + & + & 17.3 \\
\hline 2 & $\mathrm{M} / 67$ & $\mathrm{NC}$ & PD & Supraglottic & $\mathrm{T} 2 \mathrm{~N} 2 \mathrm{cM} 0$ & $\begin{array}{c}\text { TL+SND } \\
+ \text { PRT }\end{array}$ & $\begin{array}{l}8 \mathrm{R}, \\
10 \mathrm{M}, \\
15 \mathrm{DOD}\end{array}$ & + & + & + & + & 15.5 \\
\hline 3 & $\mathrm{~F} / 64$ & $\mathrm{SCC}$ & WD & Glottic & T1N0M0 & $\mathrm{LC}$ & 60 NED & _ & - & - & - & 6.8 \\
\hline 4 & $\mathrm{M} / 69$ & $\mathrm{SCC}$ & WD & Glottic & T1bN0M0 & FPL & 60 NED & + & _- & _- & + & 8.7 \\
\hline 5 & $\mathrm{M} / 63$ & $\mathrm{SCC}$ & WD & Glottic & T2N0M0 & VPL & 60 NED & - & - & - & + & 7.0 \\
\hline 6 & $\mathrm{M} / 75$ & $\mathrm{SCC}$ & WD & Glottic & T1N0M0 & $\mathrm{LC}$ & 62 NED & + & + & - & + & 8.5 \\
\hline 7 & $\mathrm{M} / 57$ & SCC & WD & Glottic & T1N0M0 & $\mathrm{LC}$ & 63 NED & - & - & - & - & 8.4 \\
\hline 8 & $\mathrm{M} / 64$ & $\mathrm{SCC}$ & WD & Glottic & T3N0M0 & TL & 60 NED & + & + & - & + & 15.0 \\
\hline 9 & $\mathrm{M} / 80$ & $\mathrm{SCC}$ & WD & Supraglottic & $\mathrm{T} 2 \mathrm{~N} 2 \mathrm{M} 0$ & $\mathrm{TL}$ & $\begin{array}{l}12 \mathrm{R}, \\
24 \mathrm{DOD}\end{array}$ & + & + & - & - & 22.0 \\
\hline 10 & $\mathrm{M} / 49$ & $\mathrm{SCC}$ & MD & Glottic & T1N0M0 & VPL & 24 NED & + & - & - & - & 7.8 \\
\hline 11 & $\mathrm{M} / 65$ & $\mathrm{SCC}$ & MD & Glottic & T3N0M0 & TL+PRT & 48 lost & - & + & + & + & 10.3 \\
\hline 12 & $\mathrm{M} / 37$ & $\mathrm{SCC}$ & PD & Supraglottic & $\mathrm{T} 2 \mathrm{~N} 1 \mathrm{M} 0$ & NPL & 14 NED & + & + & + & + & 8.3 \\
\hline 13 & $\mathrm{M} / 55$ & $\mathrm{SCC}$ & WD & Glottic & T1N0M0 & $\mathrm{LC}$ & 15 NED & - & - & - & - & 6.2 \\
\hline 14 & $\mathrm{M} / 59$ & $\mathrm{SCC}$ & MD & Glottic & T4N0M0 & TL+PRT & $11 \mathrm{DOD}$ & + & + & + & + & 10.5 \\
\hline 15 & $\mathrm{M} / 71$ & $\mathrm{SCC}$ & MD & Glottic & T2N0M0 & TL+PRT & $13 \mathrm{DOD}$ & + & + & - & + & 12.5 \\
\hline 16 & $\mathrm{M} / 38$ & $\mathrm{SCC}$ & WD & Glottic & T1N0M0 & $\mathrm{LC}$ & 19 NED & - & - & - & - & 7.3 \\
\hline 17 & $\mathrm{M} / 64$ & $\mathrm{SCC}$ & MD & Glottic & T3N0M0 & TL+PRT & 6 DOD & + & + & + & + & 18.7 \\
\hline 18 & $\mathrm{M} / 61$ & SCC & PD & Glottic & T3N0M0 & TL+PRT & 49 NED & + & + & - & + & 7.9 \\
\hline 19 & $\mathrm{M} / 67$ & $\mathrm{SCC}$ & MD & Glottic & T4N2M0 & TL+PRT & $11 \mathrm{DOD}$ & + & + & + & + & 23.9 \\
\hline 20 & $\mathrm{M} / 51$ & $\mathrm{SCC}$ & WD & Glottic & T3N1M0 & TL+PRT & 50 NED & - & - & - & - & 11.2 \\
\hline 21 & $\mathrm{M} / 65$ & $\mathrm{SCC}$ & MD & Glottic & T2N0M0 & TL+PRT & 28 DOD & - & - & - & - & 9.1 \\
\hline 22 & $\mathrm{~F} / 69$ & $\mathrm{SCC}$ & WD & Glottic & T1N0M0 & $\mathrm{LC}$ & 9 lost & - & - & - & - & 7.7 \\
\hline 23 & $\mathrm{M} / 61$ & $\mathrm{SCC}$ & WD & Supraglottic & T1N0M0 & HPL & $\begin{array}{l}24 \text { lung } M \text {, } \\
36 \text { AWD }\end{array}$ & + & - & - & - & 11.6 \\
\hline 24 & $\mathrm{~F} / 49$ & $\mathrm{SCC}$ & WD & Glottic & T1N0M0 & $\mathrm{LC}$ & 38 NED & - & - & - & - & 6.9 \\
\hline
\end{tabular}

Pt no., patient number; TNM, tumor-node-metastasis; Glut-1, glucose transporter-1; HIF, hypoxia-inducible factor; PI3K, phosphoinositide 3-kinase; p-Akt; phosphorylated-protein kinase B; SUV, standardized uptake values; NC, neuroendocrine carcinoma; SCC, squamous cell carcinoma; HG, histological grade; WD, well-differentiated; MD, moderately-differentiated; PD, poorly-differentiated; TL, total laryngectomy; FPL, frontal partial laryngectomy; VPL, vertical partial laryngectomy; HPL, horizontal partial laryngectomy; NTL, near total laryngectomy; SND, selective neck dissection; PRT, post-operative radiotherapy; LC, laryngofissure cordectomy; R, recurrence; M, metastasis; DOD, died of disease; NED, no evidence of disease; AWD, alive with disease.

\section{Results}

Patients' characteristics. The clinicopathological findings, including age, gender, site, tumor-node-metastasis (TNM) stage, pathological type, recurrence, metastasis and follow-up, are listed in Table I. Of the 24 patients, 22 (91.7\%) presented with squamous cell carcinomas and two $(8.3 \%)$ with neuroendocrine carcinomas. In total, 19 (79.2\%), four $(16.7 \%)$, and one $(4.2 \%)$ patient exhibited tumors located in the glottis, supraglottis and subglottis, respectively. All patients underwent surgery and eight $(33.3 \%)$ received post-operative radiotherapy. The average follow-up period was 33.0 months (range, 6-63 months). During the follow-up, two patients $(8.3 \%)$ were lost, three $(12.5 \%)$ developed local recurrence and two (8.3\%) developed distant metastases. At the last follow-up, 14 patients (58.3\%) remained alive. The median overall survival time (OS) was 42.3 months [95\% confidence interval (CI), 32.3-52.2 months). The three-year survival rate was $59.3 \%$.

$S U V_{\max }$ and survival analysis. No significant difference in $\mathrm{SUV}_{\max }$ was found between pathological types, TNM stages, differentiation status and tumor sites in the patients. The mean $\mathrm{SUV}_{\max }$ was 11.2 (range, 6.2-23.9). When the mean $\mathrm{SUV}_{\max }$ was defined as the cutoff, there was a significant difference in mean survival time between the higher $(\geq 11.2, n=9)$ and lower $(<11.2, \mathrm{n}=15) \mathrm{SUV}_{\max }$ subgroups (28.3 vs. 50.9 months; $\left.\mathrm{P}=0.05\right)$ (Fig. 1).

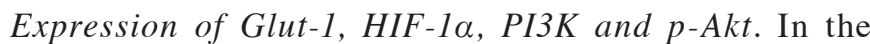
present study, 58.3\% (14/24), 50.0\% (12/24), 29.2\% (7/24) and 
B

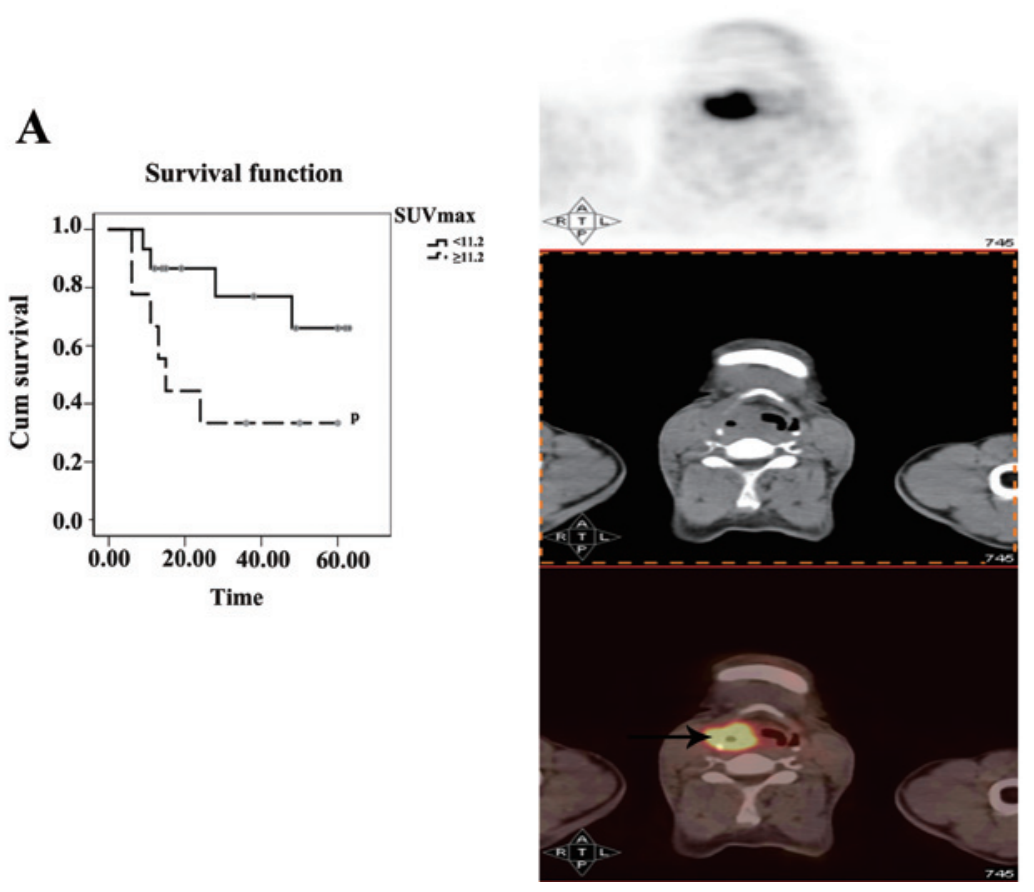

Figure 1. (A) Significant differences in mean survival time between the higher $(\geq 11.2)$ and lower $(<11.2) \mathrm{SUV}_{\max }$ subgroups were determined by univariate analysis $(\mathrm{P}=0.05)$. The multivariate analysis showed that $\mathrm{SUV}_{\max }$ was significantly associated with a poor prognosis $(\mathrm{P}=0.043)$. (B) The high $\mathrm{SUV} \mathrm{m}_{\max }$ in PET/CT. SUV, standardized uptake value; PET/CT, positron emission tomography/computed tomography.
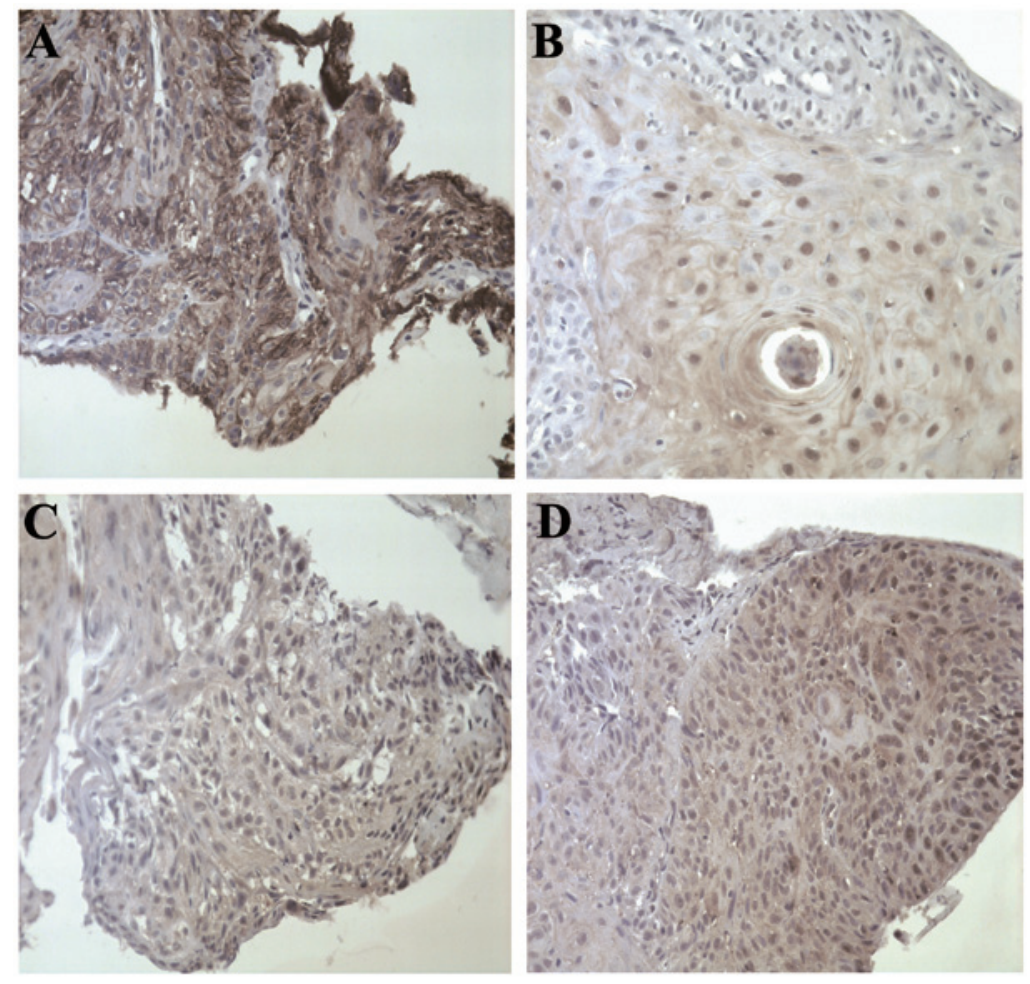

Figure 2. Immunohistochemical staining showing that (A) Glut-1 staining occurred diffusely in the membranes of the cancer cells, (B) HIF-1 $\alpha$ occurred in a granular and diffuse pattern localized mainly in the cytoplasm of the cancer cells and (C) PI3K and (D) p-AKT proteins were detected in the nucleus and cytoplasm (EliVision, x40). HIF- $\alpha$ hypoxia-inducible factor-1 $\alpha$; PI3K, phosphoinositide 3-kinase; Glut-1, glucose transporter-1; p-AKT, phosho-protein kinase B.

$54.2 \%(13 / 24)$ of the laryngeal carcinomas were positive for Glut-1, HIF-1 $\alpha$, PI3K and p-Akt protein, respectively (Table I; Fig. 2).
Spearman's rank analysis showed significant correlations between the expression of Glut-1 and HIF-1 $\alpha(\mathrm{r}=0.676$; $\mathrm{P}<0.001)$, Glut-1 and PI3K ( $\mathrm{r}=0.418 ; \mathrm{P}=0.042)$, Glut-1 and 
A

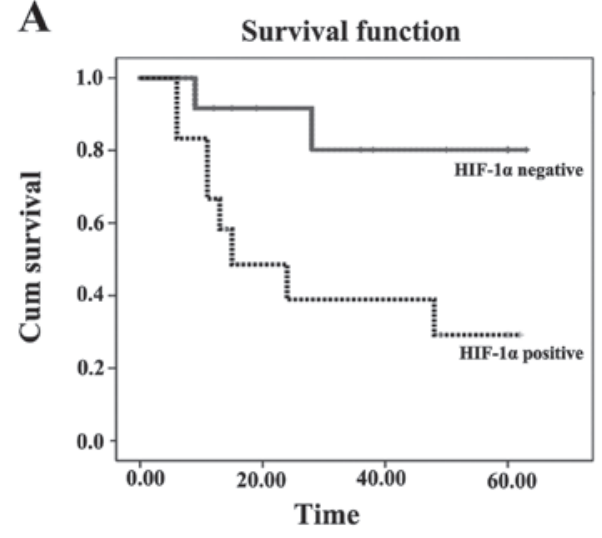

B

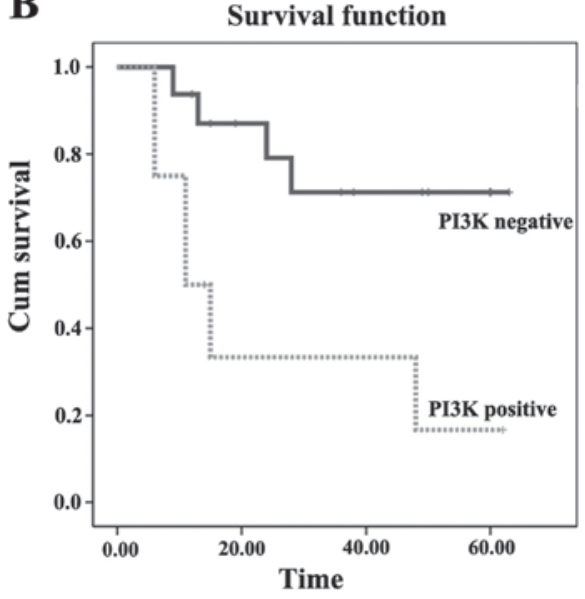

Figure 3. (A) HIF-1 $\alpha$ and (B) PI3K expression were significantly associated with a poorer survival in the univariate analyses $(\mathrm{P}=0.018$ and $\mathrm{P}=0.008$, respectively). HIF- $\alpha$, hypoxia-inducible factor-1 $\alpha$; PI3K, phosphoinositide 3-kinase.

p-Akt (r=0.580; P=0.003), HIF-1 $\alpha$ and PI3K $(r=0.707$; $\mathrm{P}<0.001), \mathrm{HIF}-1 \alpha$ and $\mathrm{p}-\mathrm{Akt}(\mathrm{r}=0.753 ; \mathrm{P}<0.001)$ and $\mathrm{PI} 3 \mathrm{~K}$ and p-Akt (r=0.650; $\mathrm{P}=0.001)$.

Correlation between $S U V_{\max }$ and hypoxic markers. Spearman's rank analysis showed significant correlations between $\mathrm{SUV}_{\max }$ and Glut-1 ( $\mathrm{r}=0.577 ; \mathrm{P}=0.003), \mathrm{HIF}-1 \alpha(\mathrm{r}=1.0 ; \mathrm{P}<0.0001)$, PI3K $(r=1.0 ; \mathrm{P}<0.0001)$ and $\mathrm{p}-\mathrm{Akt}(\mathrm{r}=0.577 ; \mathrm{P}=0.003)$ expression.

The associations between Glut-1, HIF-1 $1 \alpha$, PI3K and p-Akt expression and the clinicopathological features are shown in Table I. The univariate analyses revealed a significantly shorter survival time in those patients with high HIF-1a expression compared with negative HIF- $1 \alpha$ expression (30.4 vs. 54.5 months; $\mathrm{P}=0.018$; Fig. $3 \mathrm{~A}$ ). There was also a significant correlation between survival time and the expression of PI3K ( $\mathrm{P}=0.008$; Fig. 3B). By contrast, survival time was not correlated with Glut-1 or p-Akt expression. The multivariate analysis showed that $\mathrm{SUV}_{\max }(\mathrm{P}=0.043)$ and $\mathrm{PI} 3 \mathrm{~K}(\mathrm{P}=0.012)$ were significantly associated with a poor prognosis.

The expression of Glut-1, HIF-1 $\alpha$ and p-Akt was not significantly associated with any clinicopathological factor (gender, age, pathological type, differentiation, TNM, site, recurrence and metastasis). However, significantly higher PI3K expression was observed in poorly- and moderately-differentiated laryngeal carcinomas compared with well-differentiated carcinomas $(\mathrm{P}=0.012)$. $\mathrm{PI} 3 \mathrm{~K}$ expression was not significantly associated with the other clinicopathological factors of gender, age, pathological type, TNM, site, recurrence and metastasis.

\section{Discussion}

Previous studies have demonstrated that FDG uptake may be an independent prognostic factor in head and neck tumors in general, including for certain patients with laryngeal cancer $(3,19,27)$. In our previous study, it was found that the $\mathrm{SUV}_{\max }$ of PET/CT in cervical lymph nodes may predict cervical metastasis of carcinoma from an unknown primary tumor (25 patients) (3). In the present study, it was revealed that the mean survival time for those with a low $\operatorname{SUV}_{\max }(<11.2)$ was much longer than those with a high $\operatorname{SUV}_{\max }(\geq 11.2)$ (50.9 vs. 28.3 months, $\mathrm{P}=0.05$ ). However, the association between SUV and the prognosis of head and neck cancer continues to be debated $(28,29)$. Haerle et al did not find a significant correlation between $\mathrm{SUV}_{\max }$ and a higher prevalence of metastasis, or as a surrogate for a worse outcome (28). Schinagl et al identified that the integrated SUV was associated with local control and survival time, while $\mathrm{SUV}_{\text {mean }}$ and $\mathrm{SUV}_{\max }$ were not (29). These variations are believed to be due to use of varying treatments, a range of SUV cutoff values and the heterogeneity of tumor sites (27-29).

FDG uptake is also associated with specific molecular markers. Of these, Glut-1 plays an important role in the increased FDG uptake in cancers $(9-11,15)$. However, no correlation between FDG uptake and Glut-1 expression has been observed in patients with colorectal (16) or head and neck cancer (29). Therefore, the FDG uptake in cancer tissues may involve a complicated glucose-metabolizing pathway, and Glut-1 may not be the key factor in the pathway (16). Certain studies have revealed that other molecular markers, including the upstream regulators of Glut-1 and HIF-1 $\alpha(2,9)$, or the PI3K/Akt pathway $(15,26,30)$, are involved in the process of FDG uptake. Bos et al found that there are positive correlations between FDG uptake and Glut-1 expression, mitotic activity index, amount of necrosis, number of tumor cells/volume, expression of hexokinase I, number of lymphocytes and microvessel density (18). To the best of our knowledge, the present study is the first to evaluate the correlations between SUV and Glut-1, HIF-1 and the PI3K/Akt pathway in laryngeal carcinoma. There were significant correlations between $\mathrm{SUV}_{\max }$ and Glut-1 ( $\mathrm{r}=0.577 ; \mathrm{P}=0.003)$, HIF-1 $\alpha(\mathrm{r}=1.0 ; \mathrm{P}<0.0001)$, PI3K ( $r=1.0 ; \mathrm{P}<0.0001)$ and $\mathrm{p}-\mathrm{Akt}(\mathrm{r}=0.577 ; \mathrm{P}=0.003)$ expression, indicating that a high FDG uptake was significantly associated with a poor outcome in laryngeal carcinoma. The expression of HIF-1 $\alpha$ and PI3K was associated with survival time in the univariate analyses, and PI3K continued to be a prognostic factor in the multivariate analysis, while the other markers (Glut-1 and p-Akt) were not directly associated with survival time in patients with laryngeal carcinoma. Conversely, no significant difference was found in $\mathrm{SUV}_{\max }$ 
according to pathological type, TNM stage, differentiation or tumor site. In addition, the expression of Glut-1, HIF-1 $\alpha$, PI3K and p-Akt was not associated with these clinicopathological factors, other than PI3K being associated with poorly- and moderately-differentiated laryngeal carcinoma. Therefore, a correlation between the molecular basis of FDG uptake and these hypoxic markers was indicated; this concurs with the study by Kaira et al, which reported significant associations between FDG activity and the expression of Glut1, HIF-1 $\alpha$, hexokinase I, vascular endothelial growth factor, cluster of differentiation 34, Ki-67, mammalian target of rapamycin (mTOR) and p53 in malignant pleural mesothelioma. The PI3K/AKT/mTOR signaling pathway may play a crucial role in the glycolytic system associated with FDG uptake (15).

In the present study, the significant interdependence among Glut-1 and HIF-1 $\alpha$, and PI3K and p-Akt, indicated that Glut-1 is regulated by HIF-1 and the PI3K/Akt pathway. In our recent study, it was demonstrated that Glut-1 expression was correlated with the expression of PI3K and p-Akt in 42 patients with head and neck adenoid cystic carcinoma (31). The PI3K/Akt pathway promotes Glut-1 cell-surface trafficking and activity (32). Not only are the activation and phosphorylation of PI3K/Akt well-recognized regulators of cell growth, survival outcomes and angiogenesis, they also play significant roles in promoting glucose metabolism (33). AKT activation may be responsible for metabolic processes during the Warburg effect (15). Melstrom et al identified that PI3K inhibitors downregulated Glut-1 mRNA and protein expression, and were involved in mediating Glut-1 activity (32).

HIF-1 $\alpha$ may be involved in PI3K/Akt regulation. In addition to its role as a glucose transporter, Glut-1 is a factor in the cellular response to hypoxia as a downstream target of HIF-1 $\alpha$. The HIF complex then binds to hypoxia-responsive elements in target genes and activates their transcription. The PI3K/Akt pathway has been indicated in the control of HIF-1 $\alpha$ protein expression and Glut-1 expression $(23,33)$. Burrows et al demonstrated that the PI3K inhibitor, GDC-0941, reduced the HIF-1 $\alpha$, p-AKT and Glut-1 expression in thyroid carcinoma cells in vitro and in vivo (34).

The present study has several limitations, including the small number of patients and the variations in treatments and primary sites. Therefore, further investigation of the correlation between FDG uptake and hypoxic markers in vivo and in vitro is required.

Due to the small sample size, a definitive conclusion cannot be drawn, however, the results indicate that a high $\mathrm{SUV}_{\max }$ predicts a poor prognosis in laryngeal carcinoma. A high $\mathrm{SUV}_{\max }$ may also be associated with increased Glut-1, HIF-1 $\alpha$, PI3K and p-Akt expression. The study indicates that PET/CT can be used as a marker of tumor hypoxia and the prognosis of patients with laryngeal carcinoma. Further study is required to confirm these findings.

\section{Acknowledgements}

The authors acknowledge the National Natural Science Foundation of China (no. 81172562), and the financial support also provided by Science and Technology Department of Zhejiang Province (no. 2010C33028), the Health Department of Zhejiang Province (no. 2009A079, 2010KYA062) and the Department of Education of Zhejiang Province (no. Y200907435), China.

\section{References}

1. Dibble EH, Alvarez AC, Truong MT, Mercier G, Cook EF and Subramaniam RM: 18F-FDG metabolic tumor volume and total glycolytic activity of oral cavity and oropharyngeal squamous cell cancer: adding value to clinical staging. J Nucl Med 53: 709-715, 2012.

2. Kaira K, Okumura T, Ohde Y, et al: Correlation between 18F-FDG uptake on PET and molecular biology in metastatic pulmonary tumors. J Nucl Med 52: 705-711, 2011.

3. Zhao K, Luo XM, Zhou SH, et al: ${ }^{(18)}$ F-Fluorodeoxyglucose positron emission tomography/computed tomography as an effective diagnostic workup in cervical metastasis of carcinoma from an unknown primary tumor. Cancer Biother Radiopharm 27: 685-693, 2012.

4. Hentschel M, Paulus T, Mix M, Moser E, Nitzsche EU and Brink I: Analysis of blood flow and glucose metabolism in mammary carcinomas and normal breast: a H2(15)O PET and 18F-FDG PET study. Nucl Med Commun 28: 789-797, 2007.

5. Vander Heiden MG, Cantley LC and Thompson CB: Understanding the Warburg effect: the metabolic requirements of cell proliferation. Science 324: 1029-1033, 2009.

6. Zhou S, Wang S, Wu Q, Fan J and Wang Q: Expression of glucose transporter-1 and -3 in the head and neck carcinoma - the correlation of the expression with the biological behaviors. ORL J Otorhinolaryngol Relat Spec 70: 189-194, 2008.

7. Esen Akkas B, Gökaslan D, Güner L and Ilgin Karabacak N: FDG uptake in brown adipose tissue-a brief report on brown fat with FDG uptake mechanisms and quantitative analysis using dual-time-point FDG PET/CT. Rev Esp Med Nucl 30: 14-18, 2001.

8. Prante O, Maschauer S, Fremont V, et al: Regulation of uptake of $18 \mathrm{~F}-\mathrm{FDG}$ by a follicular human thyroid cancer cell line with mutation-activated K-ras. J Nucl Med 50: 1364-1370, 2009.

9. Han MW, Lee HJ, Cho KJ, et al: Role of FDG-PET as a biological marker for predicting the hypoxic status of tongue cancer. Head Neck 34: 1395-1402, 2012.

10. Li XF, Ma Y, Sun X, Humm JL, Ling CC and O'Donoghue JA: High 18F-FDG uptake in microscopic peritoneal tumors requires physiologic hypoxia. J Nucl Med 51: 632-638, 2010.

11. Alakus H, Batur M, Schmidt M, et al: Variable $18 \mathrm{~F}$-fluorodeoxyglucose uptake in gastric cancer is associated with different levels of GLUT-1 expression. Nucl Med Commun 31: 532-538, 2010.

12. Nagamatsu A, Umesaki N, Li L and Tanaka T: Use of $18 \mathrm{~F}$-fluorodeoxyglucose positron emission tomography for diagnosis of uterine sarcomas. Oncol Rep 23: 1069-1076, 2010.

13. Luo XM, Zhou SH and Fan J: Glucose transporter-1 as a new therapeutic target in laryngeal carcinoma. J Int Med Res 38: 1885-1892, 2010.

14. Rademakers SE, Lok J, van der Kogel AJ, Bussink J and Kaanders JH: Metabolic markers in relation to hypoxia; staining patterns and colocalization of pimonidazole, HIF-1 $\alpha$, CAIX, LDH-5, GLUT-1, MCT1 and MCT4. BMC Cancer 11: 167, 2011.

15. Kaira K, Serizawa M, Koh Y, et al: Relationship between 18F-FDG uptake on positron emission tomography and molecular biology in malignant pleural mesothelioma. Eur J Cancer 48: 1244-1254, 2012.

16. Izuishi $\mathrm{K}$, Yamamoto $\mathrm{Y}$, Sano $\mathrm{T}$, et al: Molecular mechanism underlying the detection of colorectal cancer by 18F-2-fluoro-2-deoxy-D-glucose positron emission tomography. J Gastrointest Surg 16: 394-400, 2012.

17. Robey IF, Stephen RM, Brown KS, Baggett BK, Gatenby RA and Gillies RJ: Regulation of the Warburg effect in early-passage breast cancer cells. Neoplasia 10: 745-756, 2008.

18. Bos R, van Der Hoeven JJ, van Der Wall E, et al: Biologic correlates of (18)fluorodeoxyglucose uptake in human breast cancer measured by positron emission tomography. J Clin Oncol 20: 379-387, 2002.

19. Machtay M, Natwa M, Andrel J, et al: Pretreatment FDG-PET standardized uptake value as a prognostic factor for outcome in head and neck cancer. Head Neck 31: 195-201, 2009.

20. Li LF, Zhou SH, Zhao K, et al: Clinical significance of FDG single-photon emission computed tomography: Computed tomography in the diagnosis of head and neck cancers and study of its mechanism. Cancer Biother Radiopharm 23: 701-714, 2008. 
21. Zimny M, Gagel B, DiMartino E, et al: FDG - a marker of tumour hypoxia? A comparison with [18F]fluoromisonidazole and pO2-polarography in metastatic head and neck cancer. Eur J Nucl Med Mol Imaging 33: 1426-1431, 2006.

22. Gagel B, Piroth M, Pinkawa M, et al: pO polarography, contrast enhanced color duplex sonography (CDS), [18F] fluoromisonidazole and $[18 \mathrm{~F}]$ fluorodeoxyglucose positron emission tomography: validated methods for the evaluation of therapy-relevant tumor oxygenation or only bricks in the puzzle of tumor hypoxia? BMC Cancer 7: 113, 2007.

23. Shen WQ, Cheng KJ, Bao YY, Zhou SH and Yao HT: Expression of Glut-1, HIF-1 $\alpha$, PI3K and p-Akt in a case of ceruminous adenoma. Head Neck Oncol 4: 18, 2012.

24. Shafee N, Kaluz S, Ru N and Stanbridge EJ: PI3K/Akt activity has variable cell-specific effects on expression of HIF target genes, CA9 and VEGF, in human cancer cell lines. Cancer Lett 282: 109-115, 2009.

25. Morani F, Pagano L, Prodam F, Aimaretti G and Isidoro C: Loss of expression of the oncosuppressor PTEN in thyroid incidentalomas associates with GLUT1 plasmamembrane expression. Panminerva Med 54: 59-63, 2012.

26. Fang J, Luo XM, Yao HT, Zhou SH, Ruan LX and Yan SX Expression of glucose transporter-1, hypoxia-inducible factor- $1 \alpha$, phosphatidylinositol 3-kinase and protein kinase B (Akt) in relation to [(18)F]fluorodeoxyglucose uptake in nasopharyngeal diffuse large B-cell lymphoma: a case report and literature review. J Int Med Res 38: 2160-2168, 2010.

27. Bae JS, Roh JL, Lee SW, et al: Laryngeal edema after radiotherapy in patients with squamous cell carcinomas of the larynx and hypopharynx. Oral Oncol 48: 853-858, 2012.
28. Haerle SK, Schmid DT, Ahmad N, Hany TF and Stoeckli SJ: The value of (18)F-FDG PET/CT for the detection of distant metastases in high-risk patients with head and neck squamous cell carcinoma. Oral Oncol 47: 653-659, 2011.

29. Schinagl DA, Span PN, Oyen WJ and Kaanders JH: Can FDG PET predict radiation treatment outcome in head and neck cancer? Results of a prospective study. Eur J Nucl Med Mol Imaging 38: 1449-1458, 2011

30. Tian M, ZhangH, Nakasone Y, Mogi K and Endo K: Expression of Glut-1 and Glut-3 in untreated oral squamous cell carcinoma compared with FDG accumulation in a PET study. Eur J Nucl Med Mol Imaging 31: 5-12, 2004.

31. Fang J, Bao YY, Zhou SH, et al: Recurrent prognostic factors and expression of GLUT-1, PI3K and p-Akt in adenoid cystic carcinomas of the head and neck: Clinicopathological features and biomarkers of adenoid cystic carcinoma. Oncol Lett 4: 1234-1240, 2012

32. Melstrom LG, Salabat MR, Ding XZ, et al: Apigenin inhibits the GLUT-1 glucose transporter and the phosphoinositide 3-kinase/Akt pathway in human pancreatic cancer cells. Pancreas 37: 426-431, 2008.

33. Ahn KJ, Hwang HS, Park JH, et al: Evaluation of the role of hexokinase type II in cellular proliferation and apoptosis using human hepatocellular carcinoma cell lines. J Nucl Med 50: 1525-1532, 2009.

34. Burrows N, Babur M, Resch J, et al: GDC-0941 inhibits metastatic characteristics of thyroid carcinomas by targeting both the phosphoinositide-3 kinase (PI3K) and hypoxia-inducible factor-1 $\alpha$ (HIF-1 $\alpha$ ) pathways. J Clin Endocrinol Metab 96: E1934-E1943, 2011. 